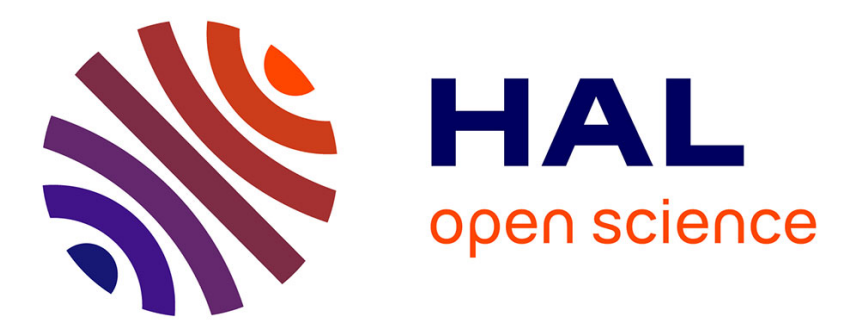

\title{
Achieving polydimethylsiloxane/carbon nanotube (PDMS/CNT) composites with extremely low dielectric loss and adjustable dielectric constant by sandwich structure
}

Benhui Fan, Yu Liu, Delong He, Jinbo Bai

\section{To cite this version:}

Benhui Fan, Yu Liu, Delong He, Jinbo Bai. Achieving polydimethylsiloxane/carbon nanotube (PDMS/CNT) composites with extremely low dielectric loss and adjustable dielectric constant by sandwich structure. Applied Physics Letters, 2018, 112 (5), pp.116 - 131. 10.1063/1.5016543 . hal01842427

\section{HAL Id: hal-01842427 \\ https://hal.science/hal-01842427}

Submitted on 3 Dec 2020

HAL is a multi-disciplinary open access archive for the deposit and dissemination of scientific research documents, whether they are published or not. The documents may come from teaching and research institutions in France or abroad, or from public or private research centers.
L'archive ouverte pluridisciplinaire HAL, est destinée au dépôt et à la diffusion de documents scientifiques de niveau recherche, publiés ou non, émanant des établissements d'enseignement et de recherche français ou étrangers, des laboratoires publics ou privés. 
Achieving polydimethylsiloxane/carbon nanotube (PDMS/CNT) composites with extremely low dielectric loss and adjustable dielectric constant by sandwich structure

\title{
Benhui FAN, Yu LIU, Delong HE* and Jinbo BAI*
}

Laboratoire Mécanique des Sols, Structures et Matériaux (MSSMat), CNRS UMR 8579, Ecole CentraleSupelec, Université Paris Saclay, Grande Voie des Vignes, 92290, Chatenay-Malabry, France

Corresponding authors: Jinbo BAI, jinbo.bai@ecp.fr

Delong HE, delong.he@ecp.fr

\begin{abstract}
Sandwich-structured composites of polydimethylsiloxane/carbon nanotube (PDMS/CNT) bulk between two neat PDMS thin films with different thicknesses are prepared by spin-coating. Taking advantage of CNT's percolation in the matrix, the composite keeps the relatively high dielectric constant $\left(\varepsilon^{\prime}=40\right.$ ) at low frequency (at $\left.100 \mathrm{~Hz}\right)$. Meanwhile, due to the existence of PDMS isolated out-layers which can limit the conductivity of the composite and consequently maintain the dielectric loss ( $\tan \delta=0.01$ ) extremely low (at $100 \mathrm{~Hz}$ ). Moreover, the same matrix of out-layer and bulk can achieve excellent interfacial adhesion and the thickness of the out-layer can be controlled by multi-cycle coating. Then based on the experimental results, the simulation works combining the percolation theory and core-shell model are employed to analyze the thickness effect of the out-layer on $\varepsilon^{\prime}$ value. Obtained relationship between the $\varepsilon^{\prime}$ of the composite and the thickness of out-layer can help to optimize sandwich structure in order to obtain the composites with adjustable $\varepsilon^{\prime}$ and extremely low $\tan \delta$.
\end{abstract}

Key words: low dielectric loss, sandwich structure 


\section{Introduction}

Polymeric matrix composites with high dielectric constant $\left(\varepsilon^{\prime}\right)$ and low dielectric loss (tan $\left.\delta\right)$ are of great interest in energy storage and harvesting devices, ${ }^{[1,2]}$ especially for capacitor's materials in ocean renewable energies. Based on the requirement of the tube-rolled structure of the devices, it requires the material not only with high dielectric performances but also maintains high flexibility in order to adapt the flow of sea waves. Polydimethylsiloxane (PDMS) is one of the candidates due to the excellent flexibility, non-toxicity to the marine environment and low cost. $^{[3-5]}$ However, PDMS is a kind of polymer with isolated nature and originally low $\varepsilon^{\prime}$ (lower than 3) which fails to meet the requirement of dielectric performance. ${ }^{[6,7]}$ Thus, developing PDMS matrix composites can be a promising alternative to solve the problem.

Generally, in light of filler's nature, the composites can be divided into two parts: one is the dielectric-dielectric (D-D) composites incorporated dielectric fillers such as ceramic fillers with high $\varepsilon^{\prime}\left(\mathrm{TiO}_{2},{ }^{[8,9]} \mathrm{ZrO}_{2},{ }^{[10,11]} \mathrm{BaTiO}_{3}(\mathrm{BT}),{ }^{[12,13]} \mathrm{CaCu}_{3} \mathrm{Ti}_{4} \mathrm{O}_{12}\right.$ (CCTO), ${ }^{[14]} \mathrm{Pb}(\mathrm{ZrTi}) \mathrm{O}_{3}(\mathrm{PZT}),{ }^{[15,16]}$ etc). The other is the dielectric-conductive (D-C) composites incorporated conductive fillers as metal particles $^{[17,18]}$ or carbon family materials (carbon black, ${ }^{[19,20]}$ carbon nanotube $(\mathrm{CNT}){ }^{[21,22]}$ graphene, ${ }^{[23,24]}$ etc). D-D composites usually need high filler contents (the volume fraction usually over $50 \%$ ) for pursuing high $\varepsilon^{\prime}$. But a high content of fillers unavoidably decreases the flexibility of polymeric matrix. D-C composites can achieve high $\varepsilon^{\prime}$ only with much lower filler's volume fraction based on the classic percolation theory, ${ }^{[25]}$ which can maintain the flexibility of the matrix. However, the dielectric loss $(\tan \delta)$ near percolation threshold $\left(f_{c}\right)$ is extremely large caused by the strong interfacial polarization. Hence, a D-C composite with low $\tan \delta(<0.05$ at 100 $\mathrm{Hz}$ ) and adjustable $\varepsilon^{\prime}(>35$ at $100 \mathrm{~Hz}$ ) will be very interesting and attractive for different applications.

Many efforts and attempts have been made to address this issue and developing conductive fillers with the core-shell structure is a popular and effective method to decrease $\tan \delta$ of the composite. ${ }^{[26]}$ Due to the existence of shell structures, it enhances the interfacial adhesion between fillers and matrix as well as impedes the leakage current in the interfacial polarization of percolation. However, fillers with core-shell structures are not easy to prepare and well control in large amounts which limits the further applications and it is still necessary to find other simple ways. Recently, designing the dielectric composite with a special structure has aroused lots of 
interest and attention. Among them, the sandwich structure is the most useful one, ${ }^{[27-31]}$ but a potential problem among them should be the strength of the interfacial adhesion between two heterogeneous materials. Furthermore, the thickness of the out-layer is hard to control which makes difficult to evaluate the influence of out-layer's thickness. Therefore, in this study, we present a sandwich-structured PDMS matrix composite with controlled out-layer thickness. The conductive filler used is CNT due to its excellent electrical conductivity and well compatibility with PDMS which can lower $f_{\mathrm{c}}$ of the composite. ${ }^{[1,35,36]}$ Then the composite with high $\varepsilon^{\prime}$ and high $\tan \delta$ is selected to be coated by thin films of PDMS respectively on sample's double surface. Due to the same matrix, the interfacial adhesion between the bulk part and out-layer is excellent after curing and the thickness of out-layer can be controlled by spin coating's condition. By adjusting the out-layer's thickness, its influence on the dielectric performance of the composite can be investigated not only by the experimental results but also by the simulation.

\section{Experimental}

\subsection{Materials:}

Liquid two-component PDMS (RT625) was provided by Wacker Chemie. The weight ratio of the siloxane based oligomer and the curing agent was 9:1. Multi-walled CNT (the commercial name: TNSM-5) was purchased from Timesnano Co. Chengdu, China. The out diameter of CNT is from 20 to $30 \mathrm{~nm}$ and the length is from 0.5 to $2 \mu \mathrm{m}$. Its electrical conductivity is over $100 \mathrm{~s} / \mathrm{cm}$ and the purity is over $98 \%$ (weight fraction).

\subsection{Fabrication of PDMS/CNT composite:}

The preparation of the composites was as follows: first, CNT with calculated weight fraction was mixed into the siloxane base oligomers by the three-roll mill for $20 \mathrm{~min}$ at room temperature ( 25 ${ }^{\circ} \mathrm{C}$ ). Then the curing agent was dropped into the slurry and mixed manually. After $30 \mathrm{~min}$ degassing in the vacuum, the slurry was removed from molds for shaping and curing by hot press at $110^{\circ} \mathrm{C}$ for $1 \mathrm{~h}$.

\section{Fabrication of the composite with multi-coating PDMS layers:}

The coating process for the selected sample was as follows: the mixture of the siloxane base oligomers and the curing agent was applied on one surface of the selected sample with the size of $10 \mathrm{~mm} \times 10 \mathrm{~mm}$. Then the coated sample was removed to a spin-coating machine to form a 
thin film on sample's surface at the speed of $7000 \mathrm{r} / \mathrm{min}$ for $2 \mathrm{~min}$. The further curing of PDMS was conducted in the oven under $110{ }^{\circ} \mathrm{C}$ for $30 \mathrm{~min}$. When the sample cooled to room temperature, another PDMS film was coated on the other face by the same method. After coating on double surfaces respectively, the sample was concerned to have one cycle's coating. The multi-cycle coating would be done for one sample in order to obtain the PDMS layer with different thickness. The whole process procedure is presented in Figure 1.

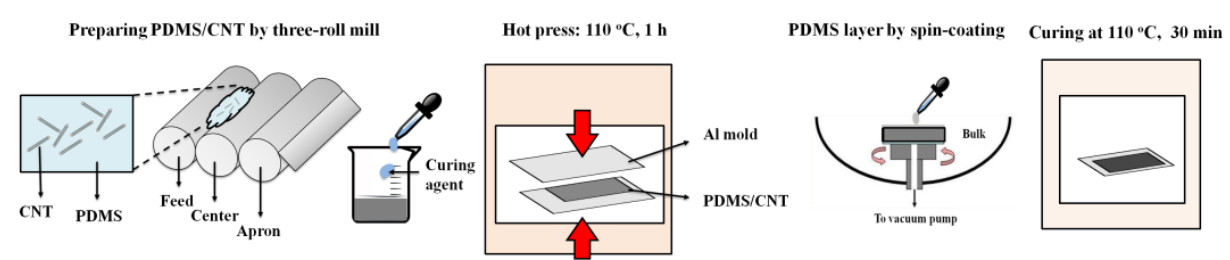

Figure 1 Schematic figure for preparation the PDMF/CNT composite with sandwich structure

\subsection{Characterization:}

The sample was tested by thickness gauge way on the iron plate (Surfix Pro, PHYNIX) to obtain different thicknesses after each coating's cycle and each time five points were tested on one plane for the average result. The dielectric properties of composites were characterized as a function of frequency by an impedance analyzer (Solartron 1260) at room temperature from $1 \mathrm{~Hz}$ to $10^{7} \mathrm{~Hz}$. After each cycle coating, the dielectric properties would be measured. Before the measurement, silver pastes were applied on both faces of the sample for reducing contacting resistance.

\section{Results and discussions}

At first, dielectric properties of samples with different CNT volume fractions are presented in Figure 2 (a) and (b), respectively. It can be found that as the volume fraction of CNT increasing, the values of $\varepsilon^{\prime}$ and $\tan \delta$ increase simultaneously which can be explained by the percolated behavior of forming micro-capacitors. ${ }^{[1,25]}$ The sample with 3.7\% CNT volume fraction is selected for the studied sample since its highest $\varepsilon^{\prime}$ and $\tan \delta$ among other samples. The thicknesses of the sample before and after multi-cycle coating measured by the thickness gauge way are listed in Table 1 and the increased thickness is calculated by subtracting with the one before coating. 


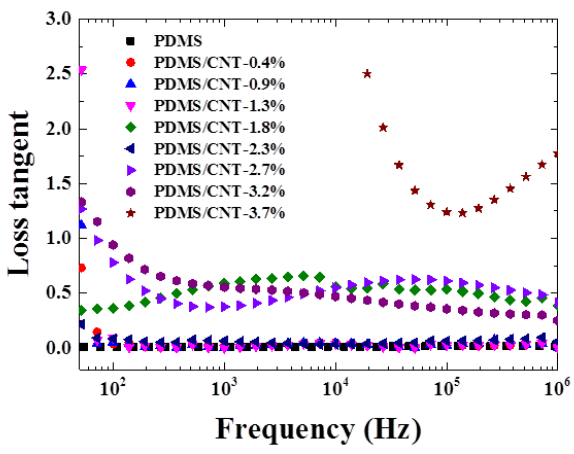

(a)

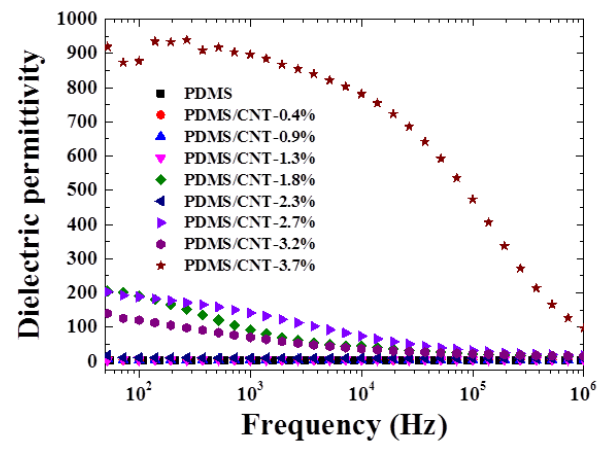

(b)

Figure 2 (a) Frequency dependence of dielectric properties with different $f_{\mathrm{CNT}}$ in the wide frequency range from $50 \mathrm{~Hz}$ to $10^{6} \mathrm{~Hz}$. (a) is for $\tan \delta$. (b) is for $\varepsilon^{\prime}$.

Table 1 Thickness of the sample before and after multi-cycle coating

\begin{tabular}{ccc}
\hline PDMS/CNT-3.7\% & Thickness of the sample $(\mathrm{d})(\mu \mathrm{m})$ & Increasing thickness $(\mu \mathrm{m})$ \\
\hline Without coating & $687.5 \pm 38.9$ & \\
$1^{\text {st }}$ coating & $800.5 \pm 48.8$ & $113 \pm 9.9$ \\
$2^{\text {nd }}$ coating & $875 \pm 40$ & $187.5 \pm 17.7$ \\
$3^{\text {rd }}$ coating & $938.5 \pm 96.9$ & $251 \pm 58$ \\
\hline
\end{tabular}

The frequency dependence of $\varepsilon^{\prime}$ and $\tan \delta$ of PDMS/CNT-3.7\% before and after multi-cycle coating are presented in Figure $3(\mathrm{a})$ and (b), respectively. Meanwhile, the values of $\varepsilon^{\prime}$ and $\tan \delta$ at $100 \mathrm{~Hz}$ and $10^{6} \mathrm{~Hz}$ are listed in Table 2 . It can be found that after multi-coating on double face of the sample, not only $\varepsilon^{\prime}$ but also $\tan \delta$ decreases significantly and the values become stable to the frequency gradually. This interesting phenomenon is attributed to the existence of the isolated PDMS layer which directly reduces the electrical conductivity $(\sigma)$ of the sample. For a sample approaching to $f_{\mathrm{c}}$, $\tan \delta$ mainly results from the conduction loss and the polarization loss of space charges, and the conduction loss contributes much more. According to the free electric theory, at low frequency, $\tan \delta$ can be expressed in Equation (1) ${ }^{[30]}$ :

$\tan \delta \approx \frac{\sigma \prime}{\omega \varepsilon_{0} \varepsilon \prime}$ 
Where $\sigma^{\prime}$ is the conductivity of the composite. The frequency dependence of AC conductivity for PDMS/CNT-3.7\% before and after multi-coating is presented in Figure 3 (c). The decrease of the conductivity for the sample after multi-cycle coating infers that coating isolated PDMS layer on sample's surface can reduce the electrical conductivity remarkably in the out-of-plane direction. Furthermore, the more the thickness increases, the more the conductivity decreases and until to a stable value. Thus, experimental results demonstrate that $\varepsilon^{\prime}$ and especially $\tan \delta$ of the composite can be controlled and reduced by multi-cycle coating isolated layer.

Table $2 \varepsilon^{\prime}$ and $\tan \delta$ for PDMS/CNT-3.7\% at $100 \mathrm{~Hz}$ and $10^{6} \mathrm{~Hz}$ before and after multi-cycle coating

\begin{tabular}{ccccc}
\hline PDMS/CNT-3.7\% & \multicolumn{2}{c}{ At $100 \mathrm{~Hz}$} & \multicolumn{2}{c}{ At $10^{6} \mathrm{~Hz}$} \\
& $\varepsilon^{\prime}$ & $\tan \delta$ & $\varepsilon^{\prime}$ & $\tan \delta$ \\
\hline Without coating & $866 \pm 14$ & $501 \pm 226$ & $112 \pm 21$ & $1.74 \pm 1.74$ \\
$1^{\text {st }}$ coating & $129 \pm 11$ & $0.08 \pm 0.03$ & $49 \pm 14$ & $0.29 \pm 0.11$ \\
$2^{\text {nd }}$ coating & $43 \pm 2.3$ & $0.04 \pm 0.03$ & $37 \pm 1.3$ & $0.16 \pm 0.02$ \\
$3^{\text {rd }}$ coating & $40 \pm 13$ & $0.01 \pm 0.004$ & $28 \pm 2.2$ & $0.08 \pm 0.02$ \\
\hline
\end{tabular}

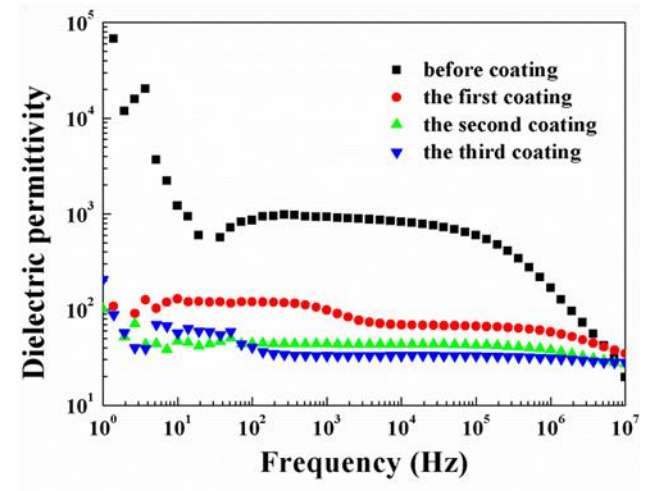

(a)

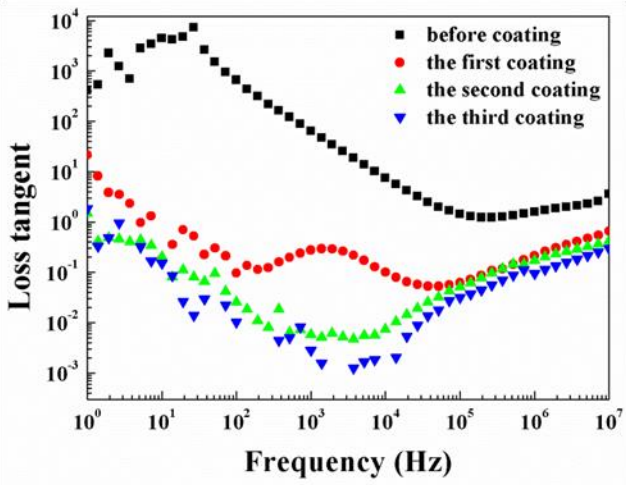

(b) 


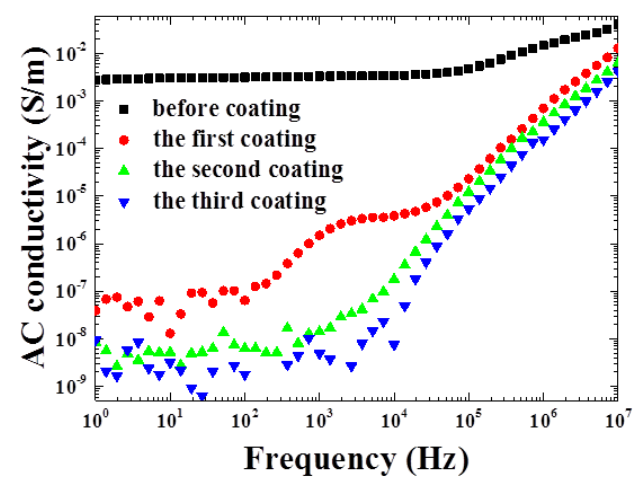

(c)

Figure 3 Frequency dependence of dielectric properties including $\varepsilon^{\prime}, \tan \delta$ and AC conductivity for PDMS/CNT-3.7\% before and after multi-cycle coating are shown in (a), (b) and (c), respectively

With the regard that coating PDMS out-layer with different thicknesses can effectively adjust $\varepsilon^{\prime}$, it will be interesting to investigate the influence of different thickness on dielectric properties for the composite by simulation. A schematic figure is presented in Figure 4 (a) to illustrate the basic structure including the dimension of the bulk material in the blue part and the thickness of the out-layer on double faces in the gray part of the sample. Meanwhile, in Figure 4 (a), the thicknesses of the out-layer by multi-coating are also presented which are $57 \mu \mathrm{m}$ after the first cycle, $94 \mu \mathrm{m}$ after the second cycle and $126 \mu \mathrm{m}$ after the third cycle, respectively. The simulation will be made based on these experimental results in the following parts.

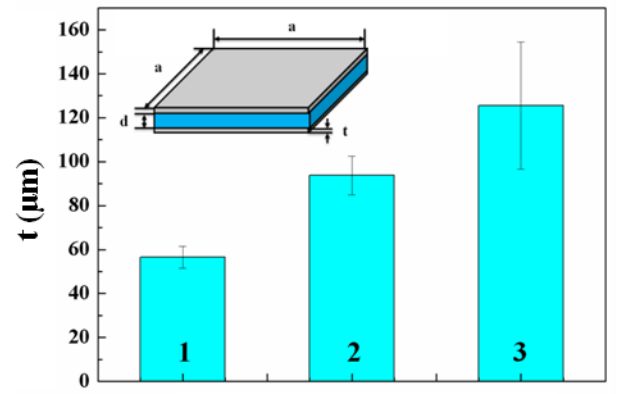

Cycle of coating

(a)

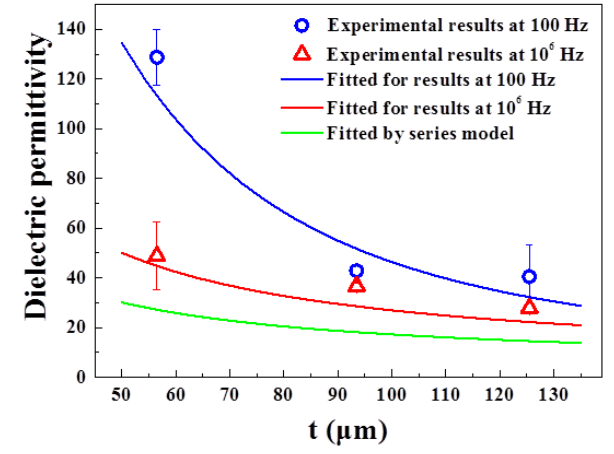

(b)

Figure 4 (a) Schematic figure of sample's structure: $d$ is the original thickness before coating: $a$ is the length and width of the sample and $t$ is the thickness of the out-layer by coating. (b) $\varepsilon^{\prime}$ of PDMS/CNT-3.7\% with different $t$ fitted by three methods 
Aforementioned, the sandwich-structured composite of the bulk part between two out-layers can be viewed as a kind of series model. Thus, the series model will be firstly used to analyze the influence of $\mathrm{t}$ on $\varepsilon^{\prime}$. The equation is presented in (2) ${ }^{[29]}$ where $\varepsilon_{\text {composite }}$ is the dielectric constant of the sandwich-structured composite; EPDMS is the one of PDMS (2.94 is used in the calculation); $\varepsilon_{\text {bulk }}$ is the one of PDMS/CNT-3.7\% before coating shown in Table 2 . The fitting result is shown in the green line of Figure $4(b)$ :

$\varepsilon_{\text {composite }}=\left(\frac{2 \mathrm{t}}{(\mathrm{d}+2 \mathrm{t}) \varepsilon_{\mathrm{PDMS}}}+\frac{\mathrm{d}}{(\mathrm{d}+2 \mathrm{t}) \varepsilon_{\text {bulk }}}\right)^{-1}$

(2)

As shown in Figure 4 (b), it can be found that the results calculated by the series model are much lower that the experimental ones, which infers that the percolation still influences $\varepsilon_{\text {composite }}$ of the composite and the influence cannot be ignored. Hence, it is necessary to retrospect the percolation theory on the dielectric response.

On the one hand, based on the polarization mechanism, the dielectric response is dependent on frequency $(f)$. For a composite with conductive fillers like $\mathrm{CNT}$, the interfacial polarization is the main contribution for $\varepsilon_{\text {composite }}$ at low frequency $(100 \mathrm{~Hz}) .{ }^{[1,25]}$ To estimate the effect of interfacial polarization, the relationship between $\varepsilon_{\text {composite }}$ and $f$ in Equation (3) ${ }^{[32]}$ is used according to percolation theory:

$\varepsilon_{\text {composite }} \propto(2 \pi f)^{\mu-1}$

where $f$ is $100 \mathrm{~Hz}$ and $\mu$ is a critical exponent. But considering the influence of $\mathrm{t}, \mu$ may be expressed as the function of $t$. In fact, the sample with the sandwich structure as shown in the schematic figure can be roughly viewed as an enlarged filler with partial core-shell structure. Meanwhile, considering that the interface volume fraction of the core-shell model can be expressed by $(4)^{[1,33]}$ :

$v=3 \frac{2 t}{d}\left[1-\frac{2 t}{d}+\frac{1}{3}\left(\frac{2 t}{d}\right)^{2}\right]$

and the coating layer does not thoroughly cover the whole sample, thus a modified interface volume fraction can be expressed by (5):

$\mu=m \cdot v$

where $m$ is defined as the relationship between $t$ and a as expressed in (6): 
$\mathrm{m}=\frac{\mathrm{a}}{\mathrm{a}+2(\mathrm{~d}+2 \mathrm{t})}$

Hence, based on these equations, $\varepsilon^{\prime}$ at $100 \mathrm{~Hz}$ of the composite can be estimated by (7)

$\varepsilon_{\text {composite }}=\varepsilon_{\text {bulk }} \cdot(2 \pi f)^{\mu-1}$

(7)

As shown in the blue line of Figure 3 (b), the simulation by modified percolation with core-shell model can well fit the experimental results of $\varepsilon^{\prime}$ at $100 \mathrm{~Hz}$. It infers that although the conductivity resulting from the interfacial polarization of bulk part can be largely suppressed by coating isolated out-layer, $\varepsilon_{\text {composite }}$ is still influenced by interfacial polarization at low frequency.

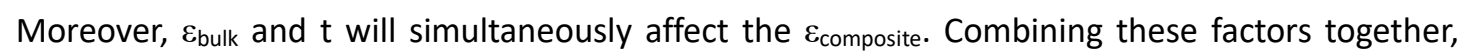
the relationship among $\varepsilon_{\text {bulk, }} \mathrm{t}$ and $\varepsilon_{\text {composite }}$ at $100 \mathrm{~Hz}$ can be obtained in Figure 5 and from the graph, we may optimize sandwich structure for designing the composite with adjustable $\varepsilon^{\prime}$ as well as extremely low $\tan \delta$.

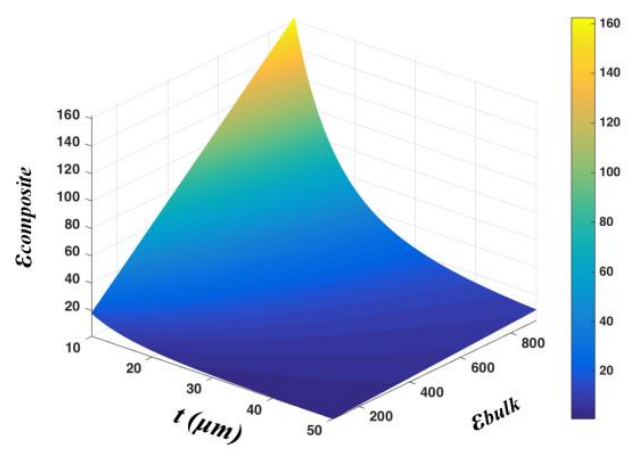

Figure 4 The relationship among t, $\varepsilon_{\text {bulk }}$ and $\varepsilon_{\text {composite. }}$

On the other hand, the polarization of $\varepsilon_{\text {composite }}$ at high frequency $\left(10^{6} \mathrm{~Hz}\right)$ is more associated with sample's structure while the interfacial polarization affects much less. Thus, for fitting $\varepsilon_{\text {composite }}$ at $10^{6} \mathrm{~Hz}$, the core-shell model in the Equation $(8)^{[34]}$ may be employed directly:

$\varepsilon=\varepsilon_{\mathrm{PDMS}}\left(1+\frac{\mathrm{d}}{\mathrm{t} * \mathrm{~m}}\right)$

(8)

The fitting result is presented in the red line of Figure 4 (b). Core-shell model can fit better than the series model which infers that the analogy for the sandwich-structured composite to 
enlarged core-shell structure is reasonable which can be explained the effect of $t$ on $\varepsilon_{\text {composite. }}$. Therefore, the modified percolation with core-shell model can be well-fitted $\varepsilon_{\text {composite }}$ at $100 \mathrm{~Hz}$ and $10^{6} \mathrm{~Hz}$. In the case of $100 \mathrm{~Hz}, \varepsilon_{\text {composite }}$ is still contributed to the interfacial polarization but PDMS out-layer can largely shield the leak current of interfacial polarization and then well control $\varepsilon_{\text {composite. }}$ Such shielding effect greatly depends on $\mathrm{t}$ and $\varepsilon_{\text {composite }}$ can be adjusted by optimizing sandwich structure with different $\mathrm{t}$. In the case of $10^{6} \mathrm{~Hz}$, the interfacial polarization influences much less on $\varepsilon_{\text {composite }}$ much less and then $\varepsilon_{\text {composite }}$ can be well fitted by core-shell model directly.

\section{Conclusion}

The existence of isolated PDMS out-layers on double faces of the PDMS/CNT composite approaching $f_{c}$ can largely decreases $\tan \delta$ by reducing the conductivity of the composite. Meanwhile, due to the percolation of CNT in the bulk part, $\varepsilon^{\prime}$ of the composite can be effectively adjusted and less frequency-dependent. Furthermore, the experimental results indicate that the thickness of the out-layer is essential to the dielectric properties. By the simulation combining percolation and core-shell model, the relationship between the thickness of out-layer and $\varepsilon^{\prime}$ of the composite can be obtained that helps to design the composite with required $\varepsilon^{\prime}$. Besides, this type of sandwich composite structure is easy to prepare for the practical applications.

\section{Reference}

[1] Zhimin Dang, Jinkai Yuan, Shenghong Yao and Ruijin Liao, Flexible nanodielectric materials with high permittivity for power energy storage, Advanced Materials, 2013, 25, 6334-6365

[2] Qin Chen, Yang Shen, Shihai Zhang and Qiming Zhang, Polymer-based dielectrics with high energy storage density, The Annual Review of Materials Research, 2015, 45, 433-58

[3] Gregor Schwartz, Zhenan Bao et.al, Flexible polymer transistors with high pressure sensitivity for application in electronic skin and health monitoring, Nature Communications, 2013, 4, 1859

[4] Evelyn K.F. Yim, Eric M. Darling, Karina Kulangara, Farshid Guilak, Kam W. Leong, 
Nanotopography-induced changes in focal adhesions, cytoskeletal organization, and mechanical properties of human mesenchymal stem cells, Biomaterials, 2010, 31, 6, $1299-1306$

[5] John J. Boland, Within touch of artificial skin, Nature Materials, 2010, 9, 790-792

[6] Jinkai Yuan, Philippe Poulin, et. al, Graphene liquid crystal retarded percolation for new high-k materials, Nature Communications, 2015, 6, 8700

[7] Stefan C. B. Mannsfeld, Zhenan Bao, et. al, Highly sensitive flexible pressure sensors with microstructured rubber dielectric layers, Nature Materials, 2010, 9, 859-864

[8] Nuoxin Xu, Liang Hu, Qilong Zhang, Xingrong Xiao, Hui Yang, and Enjie Yu, Significantly enhanced dielectric performance of poly(vinylidene fluoride-co-hexafluoropylene)-based composites filled with hierarchical flower-like $\mathrm{TiO}_{2}$ Particles, ACS Applied Materials \& Interfaces, 2015, 7, 49, 27373-27381

[9] Junjun Li, Sang, Seok, Baojin Chu, Fatih Dogan, Qiming Zhang, Qing Wang, Nanocomposites of ferroelectric polymers with $\mathrm{TiO}_{2}$ nanoparticles exhibiting significantly enhanced electrical energy density, Advanced materials, 2009, 21, 2, 217-221

[10] C. Zou, D. Kushner, and S. Zhang, Wide temperature polyimide/ $\mathrm{ZrO}_{2}$ nanodielectric capacitor film with excellent electrical performance, Applied Physics Letters, 2011, 98, 082905

[11] Neng Guo, Sara A. DiBenedetto, Pratyush Tewari, Michael T. Lanagan, Mark A. Ratner and Tobin J. Marks, Nanoparticle, size, shape, and interfacial effects on leakage current density, permittivity, and breakdown strength of metal oxide-polyolefin nanocomposites: experiment and theory, Chemistry of Materials, 2010, 22, 4, 1567-1578

[12] Benhui Fan, Junwei Zha, Dongrui Wang, Jun Zhao, Zhimin Dang, Experimental study and theoretical prediction of dielectric permittivity in $\mathrm{BaTiO}_{3} /$ polyimide nanocomposite films, Applied Physics Letter, 2012, 100,092903

[13] Benhui Fan, Junwei Zha, Dongrui Wang, Jun Zhao, Zhimin Dang, Size-dependent low-frequency dielectric properties in the $\mathrm{BaTiO}_{3} /$ poly(vinylidene fluoride) nanocomposite films, Applied Physics Letter, 2012, 100, 012903

[14] Zhimin Dang, Tao Zhou, Jinbo Bai et. al, Advanced calcium copper titanate/polyimide 
functional hybrid films with high dielectric permittivity, Advanced Materials, 2009, 21, 2077-2082

[15] Haixiong Tang, Yirong Lin, Henry A. Sodano, Enhanced energy storage in nanocomposite, capacitors through aligned PZT nanowires by uniaxial strain assembly, Advanced Energy Materials, 2012, 2, 4, 469-476

[16] Haixiong Tang, Yirong Lin, Clark Andrews and Henry A Sodano, Nanocomposites with increased energy density through high aspect ratio PZT nanowires, Nanotechnology, $2011,22,015702$

[17] Ke Yang, Xingyi Huang, Jinliang He, and Pingkai Jiang, Strawberry-like core-shell Ag@polydopamine@BaTiO 3 hybrid nanoparticles for high- k polymer nanocomposites with high energy density and low dielectric loss, Advanced Materials Interfaces, 2015, 1500361

[18] Gang Li, Shuhui Yu, Rong Sun, Daniel Lu, Clean and in-situ synthesis of copper-epoxy nanocomposite as a matrix for dielectric composites with improved dielectric performance, Composites Science and Technology, 2015, 110, 95-102

[19] Yanhui Huang and Linda S. Schadler, Understanding the strain-dependent dielectric behavior of carbon black reinforced natural rubber - An interfacial or bulk phenomenon? Composites Science and Technology, 2017, 142, 91-97

[20] Menglong Huang, Lewis B. Tunnicliffe, Jian Zhuang, Wei Ren, Haixue Yan and James J. C. Busfield, Strain-dependent dielectric behavior of carbon black reinforced natural rubber, Macromolecules, 2016, 49, 2339-2347

[21] Benhui Fan, Fahmi Bedoui, Steven Weigand and Jinbo Bai, Conductive network and $\beta$ polymorph content evolution caused by thermal treatment in carbon nanotubes- $\mathrm{BaTiO}_{3}$ hybrids reinforced polyvinylidene fluoride composites, The Journal of Physical Chemistry C, 2016, 120, 9511-9519

[22] Benhui Fan, Delong He, Yu Liu and Jinbo Bai, Influence of thermal treatments on the evolution of conductive paths in carbon nanotube- $\mathrm{Al}_{2} \mathrm{O}_{3}$ hybrid reinforced epoxy composites, Langmuir, 2016, DOI: 10.1021/acs.langmuir.6b03915

[23] Chao Wu, Xingyi Huang, Pingkai Jiang et. al, Hyperbranched-polymer functionalization 
of graphene sheets for enhanced mechanical and dielectric properties of polyurethane composites, Journal of Materials Chemistry, 2012, 22, 7010

[24] Dorina M. Opris, Martin Molberg, Christian Walder, Yee Song Ko, Beatrice Fischer and Frank A. Nüesch, New silicone composites for dielectric elastomer actuator applications in competition with acrylic foil, Advanced Functional Materials, 2011, 21, 3531-3539

[25] Cewen Nan, Yang shen, Jing Ma, Physical properties of composites near percolation, The Annual Review of Materials Research, 2010, 40, 131-51

[26] Xingyi Huang and Pingkai Jiang, Core-Shell Structured High- k Polymer Nanocomposites for energy storage and dielectric applications, Advanced Materials, 2014, DOI: 10.1002/adma.201401310

[27] Penghao Hu, Jianjun Wang, Yang Shen, Yuhan Guan, Yuanhua Lin and Ce-Wen Nan, Highly enhanced energy density induced by hetero-interface in sandwich-structured polymer nanocomposites, Journal of Materials Chemistry A, 2013, 1, 12321-12326

[28] Penghao Hu, Yang Shen, Cewen Nan et. al, Topological-structure modulated polymer nanocomposites exhibiting highly enhanced dielectric strength and energy density, Advanced functional Materials, 2014, 24, 3172-3178

[29] Moran Wang, Ning Pan, Predictions of effective physical properties of complex multiphase materials, Materials Science and Engineering R, 2008, 63 1-30

[30] Binghao Wang, Wei Zhang et. al, Two-layer materials of polyethylene and a carbon nanotube/cyanate ester composite with high dielectric constant and extremely low dielectric loss, Carbon, 2013, 54, 224-233

[31] Shenghong Yao, Jinkai Yuan, Hasan-al Mehedi, Etienne Gheeraert and Alain Sylvestre, Carbon nanotube forest based electrostatic capacitor with excellent dielectric performances, Carbon, 2017, 116, 648-654

[32] Zhimin Dang, Shenghong Yao, Jinkai Yuan and Jinbo Bai, Tailored dielectric based on microstructure change in BaTiO3-carbon nanotubes/polyvinylidene fluoride three-phase nanocomposites, The Journal of Physical Chemistry C, 2010, 114, 13204-13209

[33] T J lewis, Interfaces are the dominant feature of dielectrics at the nanometric level, IEEE Transactions on Dielectrics and Electrical Insulation, 2004, 11, 739-753 
[34] Yang Shen, Yuanhua Lin, and Cewen Nan, Interfacial effect on dielectric properties of polymer nanocomposites filled with core/shell-structured particles, Advanced Functional Materials, 2007, 17, 2405-2410

[35] Zhimin Dang, Mingsheng Zheng, and Junwei Zha. 1D/2D Carbon Nanomaterial-Polymer Dielectric Composites with High Permittivity for Power Energy Storage Applications. Small, 2016, 12, 1688-1701.

[36] Zhimin Dang, Jinkai Yuan, Junwei Zha, Tao Zhou, Shengtao Li, Guohua Hu, Fundamentals, processes and applications of high-permittivity polymer matrix composites, Progress in materials Science, $2012,57,660-723$ 